\title{
Wnt3a/ $\beta$-Catenin/CBP Activation in the Progression of Cervical Intraepithelial Neoplasia
}

\author{
Dingqing Feng ${ }^{1}$, Jie Lin ${ }^{2}$, Wenhui Wang ${ }^{1,3}$, Keqin Yan ${ }^{1}$, Haiyan Liang ${ }^{1}$, Jing Liang ${ }^{1}$, Huan Yu ${ }^{1}$ \\ and Bin Ling ${ }^{1,3 *}$
}

${ }^{1}$ Department of Obstetrics and Gynecology, China-Japan Friendship Hospital, Beijing, China, ${ }^{2}$ Department of Pathology, ChinaJapan Friendship Hospital, Beijing, China, ${ }^{3}$ Chinese Academy of Medical Sciences and Peking Union Medical College, Beijing, China

OPEN ACCESS

Edited by: László Kopper,

Semme/weis University, Hungary

*Correspondence:

Bin Ling

lingbin.ling@vip.sina.com

Received: 23 September 2020

Accepted: 10 February 2021

Published: 31 March 2021

Citation:

Feng D, Lin J, Wang W, Yan K, Liang H,

Liang J, Yu H and Ling B (2021)

Wnt3a/ $\beta$-Catenin/CBP Activation in

the Progression of Cervical

Intraepithelial Neoplasia.

Pathol. Oncol. Res. 27:609620.

doi: 10.3389/pore.2021.609620
Piwil2 reprograms HPV-infected reserve cells in the cervix into tumor-initiated cells (TICS) and upregulates Wnt3a expression sequentially, which leads to cervical intraepithelial neoplasia (CIN) and ultimately squamous cell carcinoma (SCC). However, little is known regarding Wnt signaling in the maintenance of TIC stemness during the progression of cervical lesions. We herein investigated the expression of canonical Wnt3a signaling and related genes by microarray data set analysis and immunohistochemical $(\mathrm{IHC})$ staining of samples obtained by biopsy of normal cervix, low- and high-grade CIN, and invasive SCC tissue. Array data analyzed by GEO2R showed higher expression levels of Wnt signaling and their target genes, significant upregulation of stemness-associated markers, and notably downregulated cell differentiation markers in CIN and SCC tissues compared with those in the normal cervix tissue. Further, Gene Set Enrichment Analysis (GSEA) revealed that Wnt pathway-related genes significantly enriched in SCC. IHC staining showed gradually increased immunoreactivity score of Wnt3a and CBP and notable translocation of $\beta$-catenin from the membrane to the cytoplasm and nucleus during the lesion progression. The intensity and proportion of P16, Ki67 and CK17 staining also increased with the progression of cervical lesions, whereas minimal to negative Involucrin expression was observed in CIN2/3 and SCC. Therefore, canonical Wnt signaling may contribute to the progression of $\mathrm{CIN}$ to SCC and may be a potential therapeutic target.

Keywords: cervical intraepithelial neoplasia, wnt signaling, CBP, Piwil2, stemness, Differentiation

\section{INTRODUCTION}

Cervical cancer is among the most preventable human malignancies, yet it remains a leading cause of death among women worldwide, accounting for more than 300,000 deaths annually [1]. In 2015, an estimated 98,900 new cases of invasive cervical cancer were diagnosed, and 30,500 women in China died from this cancer [2]. High-risk human papillomavirus (HR-HPV) is the major cause of cervical cancer, and in the precursor stages of cervical intraepithelial neoplasia (CIN, graded 1-3 according to severity), viral oncoprotein E6 and E7 targeting and degradation of P53 and retinoblastoma protein $(\mathrm{pRb})$ lead to cell transformation, telomerase activation, and immortalization of the infected cells [3]. Further, it has been reported that E6 and E7 initiate the expression of Piwil2, which reactivates multiple germline factors of $c-M y c$, Klf4, Sox2, Nanog, and Oct4 and reprograms HR-HPV-infected cells to tumor-initiating cells (TICs) [4]. The regulation of TIC stemness and differentiation plays a 
pivotal role in the progression of CIN to invasive cancer. However, the precise mechanism remains to be determined.

Wnts are secreted cysteine-rich glycoproteins that act as shortrange ligands to locally activate receptor-mediated signaling pathways $[5,6]$. A key step in the activation of Wnt target genes is the formation of a complex of $\beta$-catenin with members of the T-cell transcription factor (TCF)/lymphoid enhancer-binding factor (LEF) family of transcription factors [6-8]. To generate a transcriptionally active complex, TCF/ $\beta$-catenin recruits the KAT3 transcriptional coactivator CREBbinding protein (CBP) or its closely related homolog, p300, as well as other components of the basal transcription machinery, to initiate transcription $[6,9,10]$. As Kahn and colleagues reported, $\mathrm{CBP} / \beta$-catenin-mediated transcription is essential for stem and/ or progenitor cell maintenance and proliferation, whereas a switch to $\mathrm{p} 300 / \beta$-catenin-mediated transcription is a critical step in the initiation of differentiation and a decrease in cellular potency [6]. In addition to regulating cellular processes, including proliferation, differentiation, motility, and survival, the canonical $\beta$-catenin-dependent Wnt signaling pathway plays crucial roles in embryonic development and maintenance of homeostasis in mature tissues [11, 12]. Wnt signaling is also involved in stem cell maintenance by modulating the levels of intrinsic pluripotency factors, for example, Oct4, Nanog, and Sox2 [13-15], and Wnt3a supplementation has been demonstrated to maintain the pluripotency of embryonic stem cells $[16,17]$.

Accumulating evidence suggests that the Wnt/ $\beta$-catenin signaling pathway is often involved in oncogenesis and cancer development $[6,11,18]$. The importance of aberrant Wnt signaling in some types of cancer, such as colorectal and breast cancer, is clear. In mice, it has long been known that misexpression of Wnt-1, -3, or -10 induces mammary adenocarcinomas $[19,20]$. The $\operatorname{APC}^{\mathrm{Min}}$ mouse has also been shown to exhibit an enhanced incidence $(\sim 10 \%)$ of spontaneous mammary cancer and a greatly increased susceptibility (90\%) to carcinogen-induced mammary cancer [21, 22]. However, aberrant Wnt signaling may play a role in many other types of malignancies, even those without the classical mutations associated with the pathway. Our previous study reported that the HPV16 oncoproteins E6 and E7 reprogrammed HaCaT cells and led to TIC formation by restoring Piwil2 expression [4]. We also observed that Piwil2 synchronously activated Wnt3a signaling to enhance reprogramming and maintain the stemness of TICs via the $\mathrm{CBP} / \beta$-catenin interaction [data unpublished]. To explore the potential role of $\mathrm{Wnt} 3 \mathrm{a}$ in the progression of cervical neoplasia, we investigated the expression of canonical Wnt3a signaling and related genes with microarray data set analysis and immunohistochemical staining of biopsied samples of normal cervix, low- and high-grade cervical intraepithelial neoplasia (CIN), and invasive cervical squamous cell carcinoma (SCC) tissue.

Our present findings provide novel pathological insights into Wnt3a signaling in the progression of CIN and aggressiveness of cervical cancer, thus defining a potential therapeutic target for cervical lesions.

\section{MATERIALS AND METHODS}

\section{Clinical Samples}

The project was approved by the Ethics Committee of ChinaJapan Friendship Hospital (approval number: 2020-28-K20). One hundred thirty-four histological specimens, including hysterectomy tissues and loop/cone biopsied samples, were retrieved from the files of the Department of Pathology of China-Japan Friendship Hospital. The specimens consisted of normal cervix $(n=20)$, CIN1 $(n=36), \operatorname{CIN} 2 / 3(n=32)$, and invasive SCC $(n=46)$ tissues.

\section{Immunohistochemistry Analysis}

Four-micrometer-thick formalin-fixed, paraffin-embedded tissue sections were deparaffinized with xylene and rehydrated through a graded series of ethanol. Antigen retrieval was performed with $0.01 \mathrm{M}$ citrate buffer ( $\mathrm{pH}$ 6.0) in a microwave oven for $10 \mathrm{~min}$. After cooling to room temperature, the slides were incubated with $3 \% \mathrm{H}_{2} \mathrm{O}_{2}$ for $10 \mathrm{~min}$ to inactivate endogenous peroxidase. The slices were incubated overnight at $4^{\circ} \mathrm{C}$ in a humidified chamber with the following primary antibodies: 1:500 diluted anti-Wnt3a (ab28472), 1:500 diluted anti- $\beta$-catenin (ab32572), 1:1000 diluted anti-CBP (ab50702), 1:100 diluted anti-cytokeratin 17 (CK 17, ab53707), 1:1000 diluted anti-involucrin (ab53112), 1:200 diluted anti-CDKN2A/p16INK4a (p16, ab54210), and 1:200 diluted antiKi67 (ab16667). All of the primary antibodies were purchased from Abcam, United States. The binding of the primary antibodies was visualized using the ChemMate detection kit (PV-9000, ZSBIO, China). The slices were lightly counterstained with Mayer's hematoxylin for $30 \mathrm{~s}$.

Immunoreactivity was semiquantitatively evaluated. The degree of positive staining for all antibodies was evaluated by score on a scale of $0-3$ for strength of intensity of staining and on a scale of $0-4$ for percentage of positive cells. The final total score was generated by the intensity score $x$ proportion score. The intensity score was defined as follows: 0 , no staining of epithelial cells; 1 , weak staining; 2 , moderate staining; or 3, strong staining. The proportion score was defined as follows: 0 , no staining of the cervical epithelial cells in any field; $1,<10 \%$ of the epithelium stain-positive; $2,11-50 \%$ stain-positive; $3,51-80 \%$ stain-positive; or $4,>80 \%$ stain-positive. The total score ranged from 0 to 12 . Two different pathologists evaluated all the specimens in a blinded manner. Notably, the distribution of staining in the intraepithelial lesions (CIN1 and CIN2/3) was compared with that of the normal cervical squamous epithelium. CIN tissues were categorized as having the thickness of the samples being from two-thirds to fully stained.

\section{Microarray and Gene Set Enrichment Analysis}

To investigate the expression pattern of genes associated with the activation of $\mathrm{Wnt} / \beta$-catenin signaling in the progression of cervical lesions, the data set GSE64217 (containing 2 normal cervix, $2 \mathrm{CIN} 2 / 3$, and 2 cervical squamous cell cancer samples), GSE51993 (containing 7 HPV- normal cervix samples, 9 HPV + 

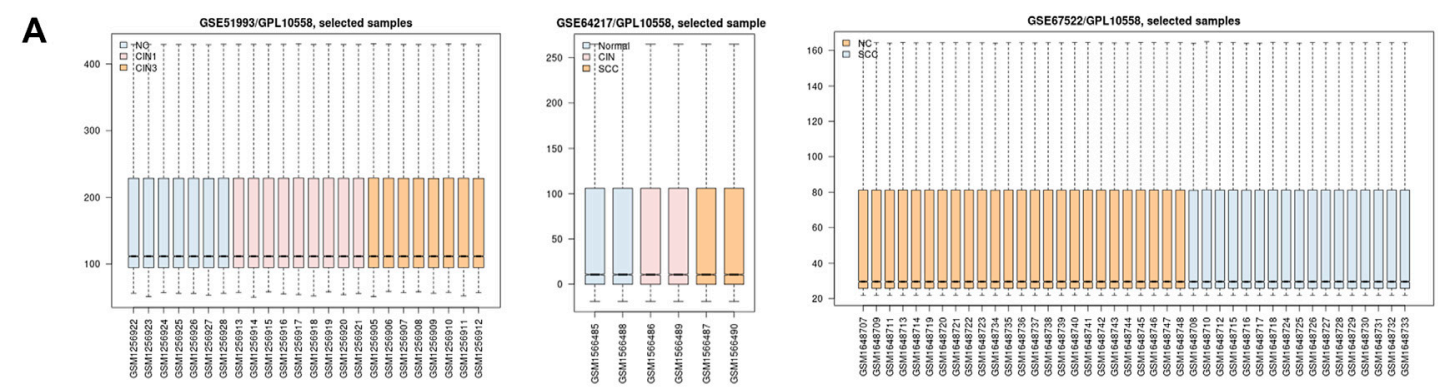

B
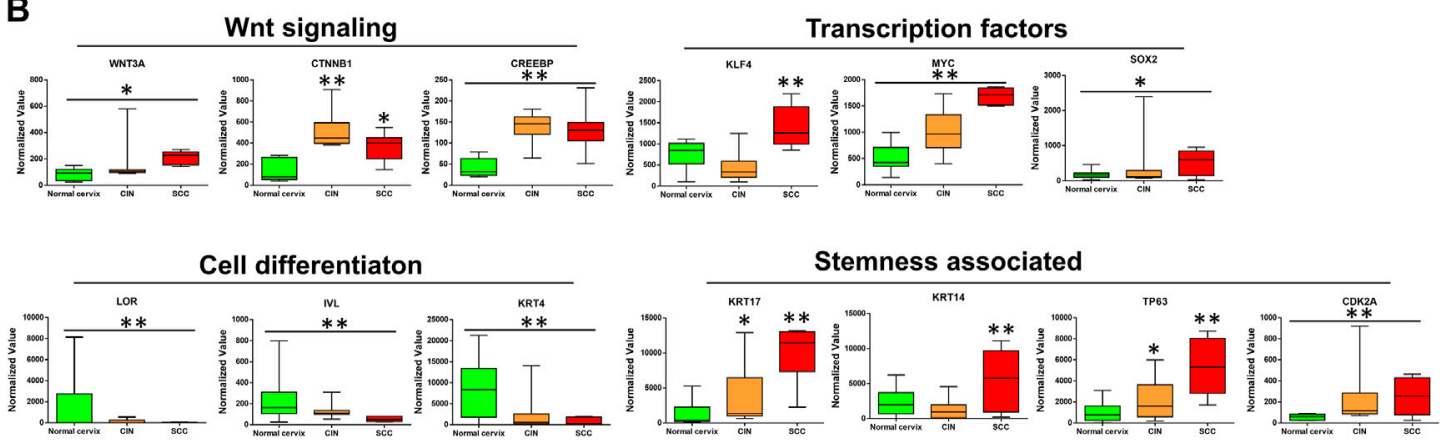

FIGURE 1 | The bioinformatics analyses of the microarray data sets of GSE64217, GSE51993 and GSE67522 containing normal cervix, CIN, and SCC sample information (A) Boxplot of the distribution of normalized expression values (B) The microarray data sets of GSE64217, GSE51993 and GSE67522 were analyzed by GEO2R on GEO database. Wnt3a/CTNNB1/CREBBP and their target genes MYC and SOX2 showed higher expression levels in CIN and SCC than in normal cervix. The stemness-associated markers were significantly upregulated, while the cell differentiation markers were notably downregulated in CIN and SCC. The expression values of KLF4 and CK14 decreased moderately in CIN as compared with normal cervix. CIN and SCC compared with normal cervix, ${ }^{*} p<0.05,{ }^{* *} p<0.01$.

CIN I samples and $8 \mathrm{HPV}+$ CIN III samples), GSE67522 (containing 11 HPV16- normal cervix samples, 11 HPV16+ non-malignants and $20 \mathrm{HPV} 16$ + cervical cancer tissues) were downloaded from the GEO database (https://www.ncbi.nlm.nih. gov/geo/). The platforms for these data sets are all based on GPL10558 and an Illumina HumanHT-12 V4.0 Expression BeadChip. The downloaded files were processed using the $\mathrm{R}$ package and then calibrated, standardized, and converted to log2 data, or normalized with cubic spline method. The limma package was used to standardize the gene chip and analyze the differential expression. The quantile normalized values for canonical Wnt signaling pathway associated genes were found out via VLOOKUP from the gene expression description tables. Boxplot was used to show the expression difference among groups of normal cervix, CIN and SCC.

\section{Statistical Analyses}

The data are presented as the means \pm SD. Statistical analyses were performed with SPSS version 25 (SPSS Inc., Chicago, IL, United States). The significance of gene expression between groups in microarray data sets were analyzed with one-way analysis of variance. The comparison of immunoreactivity score between normal cervix and cervical lesions were performed with two-tailed Chi-square test. Pearson test was used to analyze the correlation between the expression of the biomarkers and the cervical lesion degree. A $p$ value $<0.05$ was considered significant.

\section{RESULTS}

\section{Expression Pattern of Canonical Wnt Pathway-Related Genes}

To identify the differentially expressed genes (DEGs), the data set GSE64217, GSE51993 and GSE67522 were analyzed by the GEO2R method on the GEO website. The boxplot of the distribution of the normalized expression values for selected samples demonstrates that the data were suitable for the analysis and "limma" (Figure 1A), which was used to perform a top table computation to extract a list of the top-ranked genes. Within the top 250 differentially expressed genes, the canonical Wnt signaling genes Wnt $3 a, C T N N B 1$ ( $\beta$-catenin), and CREBBP $(C B P)$ were significantly upregulated in the samples of precancerous and cancer of the cervix compared with those of the normal cervix, thereby contributing to the distinct upregulation of their downstream transcription factors KLF4, $M Y C$, and SOX2, except KLF4 slightly downregulated in CIN (Figure 1B). As previously reported, $\mathrm{CBP} / \beta$-catenin signaling is important for the maintenance of stem cell pluripotency [6]. KRT17 (CK17), TP63 and CDK2A (P16), markers of HPV target cells/cervical cancer stem cells (CSCs), were apparently upregulated in CIN and SCC. In contrast, markers of squamous cell differentiation, LORICRIN, INVOLUCRIN and KRT4 (CK4), were significantly downregulated in CIN and SCC (Figure 1B). It was somewhat unusual that KRT14 (CK14) and KLF4, showed in these GSE gene sets, were 
A
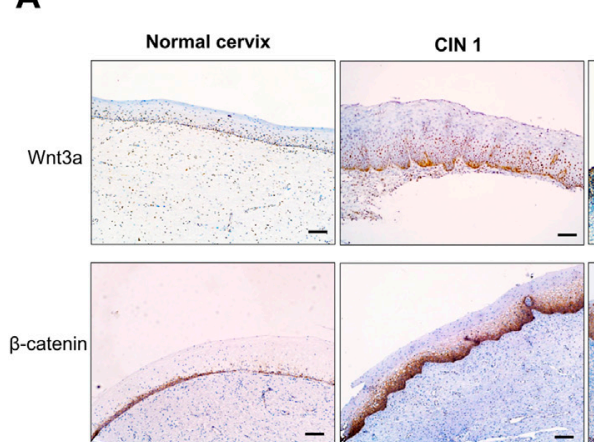

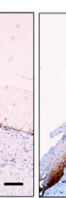
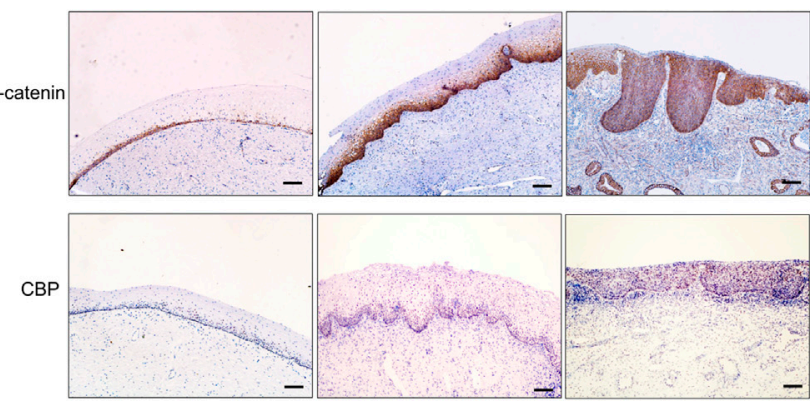

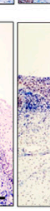

$\operatorname{CIN} 2 / 3$
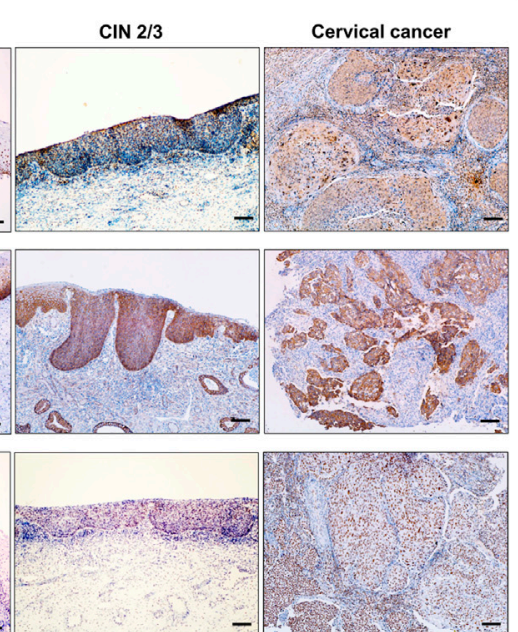

B
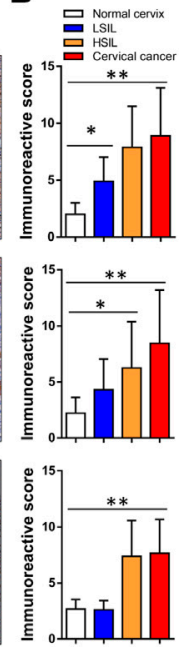

FIGURE 2 | Immunohistochemical expression pattern of Wnt3a, $\beta$-catenin and CBP in normal cervix, CIN1, CIN2/3, and SCC tissue. In the normal cervix, Wnt3a-, $\beta$-catenin- and CBP-positive cells scattering was confined to the basal cell layer. The immunoreactivity score of Wnt3a and CBP gradually increased, and $\beta$-catenin staining showed a notable positive translocation from the membrane to the cytoplasm and nucleus during the progression from CIN1 to SCC (A). The total scores of Wnt3a, $\beta$-catenin and CBP immunoreactivity were significantly higher in CIN2/3 and cervical cancer tissues compared to the normal cervix and CIN1 tissues, respectively (B). ${ }^{\star} p<0.05,{ }^{\star *} p<0.01$; Bar, $100 \mu \mathrm{m}$

TABLE 1 | Relationship between Wnt3a/ $\beta$-catenin/CBP and lesion grade of cervical squamous epithelium ( $n=134)$.

\begin{tabular}{|c|c|c|c|c|c|c|c|c|c|c|}
\hline \multirow{2}{*}{$\begin{array}{l}\text { Pathologic } \\
\text { diagnosis }\end{array}$} & \multirow[t]{2}{*}{$N$} & \multicolumn{3}{|c|}{ Wnt3a } & \multicolumn{3}{|c|}{$\beta$-catenin } & \multicolumn{3}{|c|}{ CBP } \\
\hline & & $\begin{array}{c}\text { Positive }^{a} \\
(\%)\end{array}$ & $\chi^{2}$ & $p$ & $\begin{array}{c}\text { Positive }^{a} \\
\text { (\%) }\end{array}$ & $\chi^{2}$ & $p$ & $\begin{array}{c}\text { Positive }^{a} \\
(\%)\end{array}$ & $\chi^{2}$ & $p$ \\
\hline Normal cervix & 20 & 0 & $69.263^{b}$ & $<0.01^{b}$ & $1(5 \%)$ & $40.504^{b}$ & $<0.01^{b}$ & 2 (10\%) & $21.484^{b}$ & $<0.01^{b}$ \\
\hline CIN1 & 36 & $16(44.4 \%)$ & $32.438^{c}$ & $<0.01^{\mathrm{C}}$ & $18(50 \%)$ & $11.735^{\mathrm{c}}$ & $<0.01^{\mathrm{c}}$ & $18(50 \%)$ & $7.195^{\mathrm{C}}$ & $<0.05^{\mathrm{C}}$ \\
\hline CIN2/3 & 32 & $26(81.3 \%)$ & & & 23 (71.9\%) & & & $20(62.5 \%)$ & & \\
\hline Cervical cancer & 46 & $45(97.8 \%)$ & & & 39 (84.8\%) & & & 36 (78.3\%) & & \\
\hline
\end{tabular}

${ }^{a}$ Positive staining surpassed the basal cells.

${ }^{b}$ Chi square test among groups of normal cervix, CIN1, CIN2/3, and cervical cancer.

${ }^{c}$ Chi square test among lesion groups.

moderately downregulated in CIN tissues compared with normal cervix, nevertheless they were all significantly upregulated in SCC tissues (Figure 1B).

\section{Wnt3a/ $\beta$-Catenin/CBP Expression and Cervical Lesion Grade}

In normal cervical squamous epithelium, Wnt3a staining was confined to the basal cells, whereas in CIN1 diffuse immunoreactivity was limited to the lower one-third of the epithelial layer, and in CIN2/3, the staining extended through the full thickness of the epithelial layer (Figure 2A). Strong to moderate Wnt3a immunoreactivity was observed in $45(97.8 \%)$ of 46 invasive SCCs and 26 (81.3\%) of 32 CIN2/3, while $44.4 \%$ (16/ 36) CIN1 showed light-to-moderate expression, as occasional scattered staining was observed within basal cells in normal cervix that was considered negative (Table $\mathbf{1}$ ).

A gradual increase in the $\beta$-catenin score and its positive translocation from the membrane to the cytoplasm and finally to the nucleus was noted in cervical lesions at higher stages (Figure 2A). Only 5\% (1/20) of the normal cervix samples were membrane positive for $\beta$-catenin in the parabasal cells, while one-half $(18 / 36)$ of the CIN1 samples were membrane positive for $\beta$-catenin. Approximately $71.9 \%$ (23/32) of the CIN2/ 3 and $84.8 \%(39 / 46)$ of the SCC samples had cytoplasmic and even nuclear positive staining. The pattern of CBP expression in each stage of cervical lesion was similar to that of $\beta$-catenin with only nuclear immunoreactivity (Table 1). Overall, the total scores of Wnt3a, $\beta$-catenin and CBP immunoreactivity were significantly higher in $\mathrm{CIN} 2 / 3$ and cervical cancer tissues compared to the normal cervix and CIN1 tissues, respectively (Figure 2B, $p<0.01$ ).

\section{Wnt Signaling Activation Promotes Lesion Progression}

Markers useful in the assessment of lesion grade were further observed. P16, which is valuable in the diagnosis of high-grade 


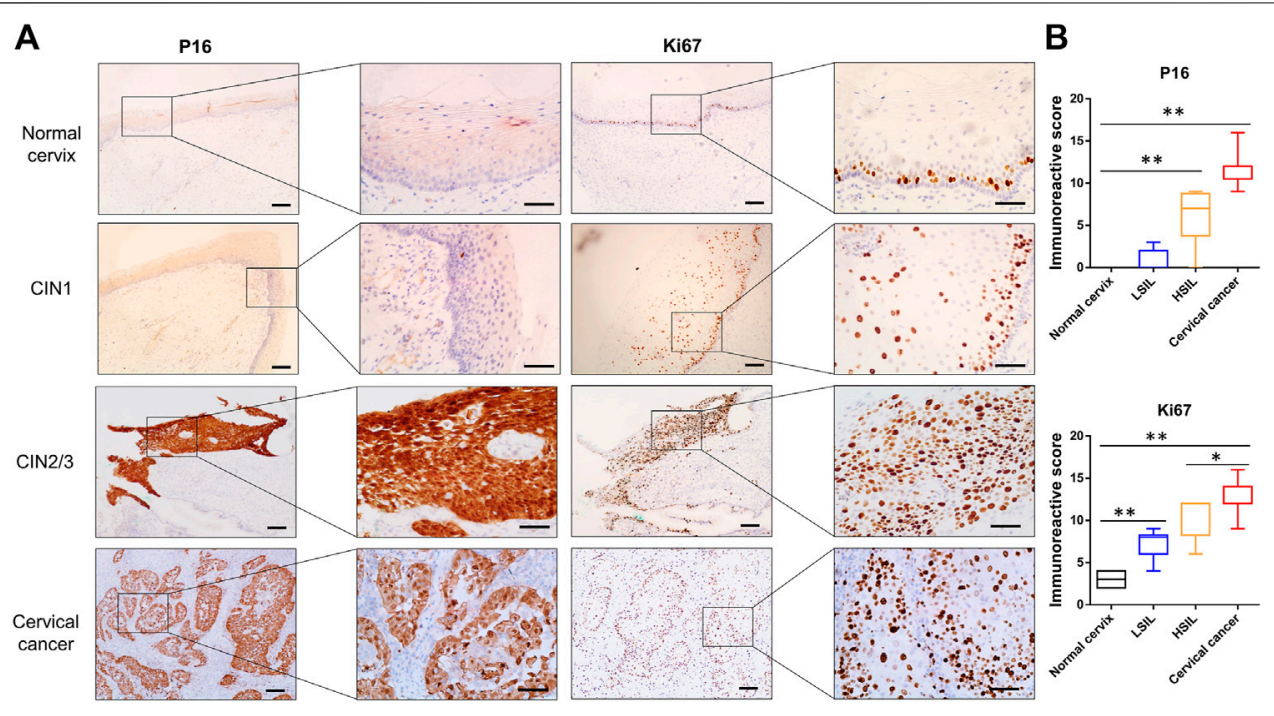

FIGURE 3 | Representative images of P16 and Ki67 immunostaining in normal cervix, CIN1, CIN2/3, and SCC tissue (A) P16 was negative in the normal cervix and CIN1 samples. Scattered Ki67 positivity was confined to the basal cells in the normal cervix but in both the basal and parabasal cells in the CIN1 samples. Diffuse immunoreactivity of P16 and Ki67 was distributed in the SCC and full thickness of the CIN2/3 samples (B) The IHC scores of P16 and Ki67 were significantly higher in both the CIN2/3 and SCC tissues compared to the CIN1 and normal cervix. ${ }^{\star} p<0.05,{ }^{\star \star} p<0.01$; Bar, $100 \mu \mathrm{m}$.
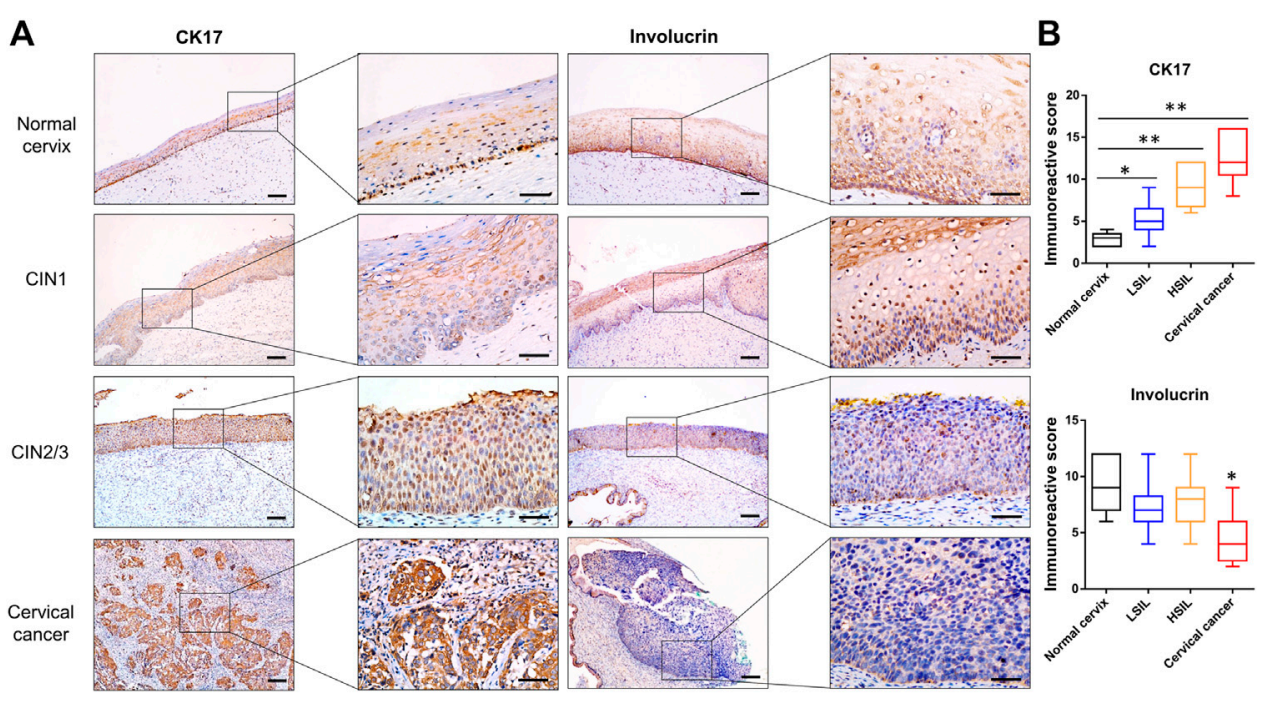

FIGURE 4 | Representative images of CK17 and Involucrin immunostaining in normal cervix, CIN1, CIN2/3, and SCC tissues (A) The reserve cells in the basal layer were positive for CK17 in the normal cervix. The full thickness of the CIN2/3 and SCC samples showed CK17 staining, whereas only a very small number of positive cells were scattered in the lower one-third of the squamous epithelium in the CIN1 samples. In contrast to the pattern of CK17 expression, diffuse immunoreactivity of Involucrin was observed in the full thickness of the normal cervix and CIN1 samples, whereas minimal and negative Involucrin expression was observed in the CIN2/3 and SCC tissue (B) The IHC score of CK17 increased gradually in accord with the lesion grade, while the score of Involucrin staining was significantly lower in SCC than that in normal cervix and CIN lesions. ${ }^{*} p<0.05,{ }^{\star *} p<0.01$; Bar, $100 \mu \mathrm{m}$.

CIN, was positively stained in the upper two-thirds and full thickness of the epithelium in most cases of CIN2/3 and in all of cervical cancer tissues, while minimal or negative P16 was observed in cervical squamous epithelium of the normal cervix or CIN1 samples (Figure 3A). As the Ki67 antigen is expressed in all active phases of the cell cycle, diffuse immunoreactivity was distributed throughout SCC and the full thickness of the CIN2/3 samples, while scattered positive staining was confined to only the lower one-third of the CIN1 and the basal cells in the normal cervix samples (Figure 3A). Statistical analysis showed that the IHC scores of P16 and Ki67 were significantly higher in both the CIN2/3 and SCC 

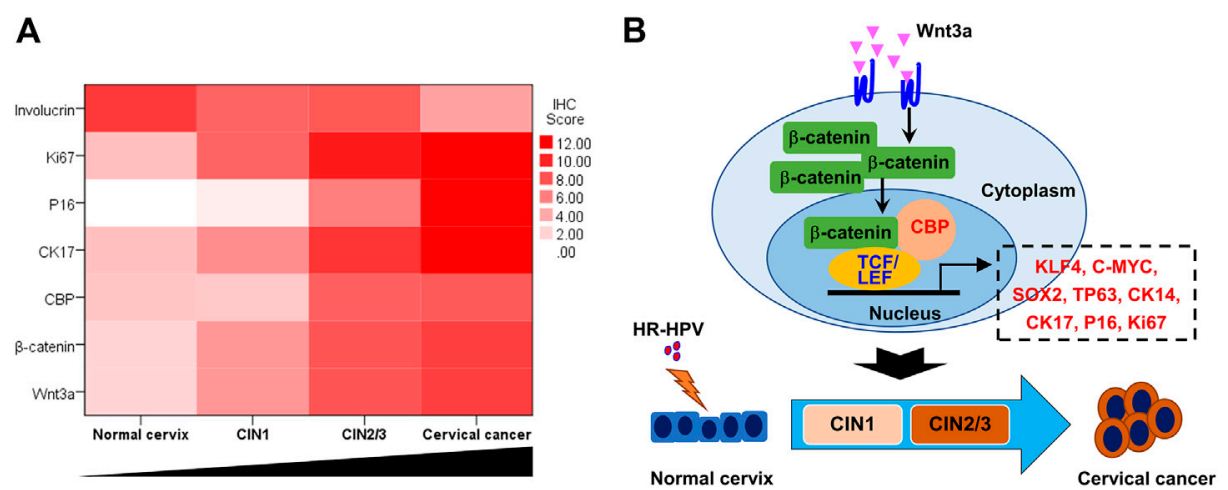

FIGURE 5 | Wht signaling pathway activation and cervical lesion progression (A) The heat map, which was made with the total IHC scores of various biomarkers and the cervical lesion grade, apparently showed the trends of Wnt/ 3 -catenin/CBP-regulated gene expression in the process of cervical lesion (B) Model of Wnt3a activation promoting the transition from HR-HPV-infected cervix to cervical cancer.

TABLE 2 | Correlation between the expression of various biomarkers and the cervical lesion grade.

\begin{tabular}{|c|c|c|c|c|c|c|c|}
\hline & \multicolumn{7}{|c|}{ Total immunoreactivity scores } \\
\hline & Wnt3a & $\beta$-catenin & CBP & P16 & Ki67 & CK17 & Involucrin \\
\hline \multicolumn{8}{|l|}{ Cervical lesion grade } \\
\hline Correlation coefficient & 0.679 & 0.577 & 0.688 & 0.898 & 0.892 & 0.867 & -0.557 \\
\hline$p$ Value & $<0.01$ & $<0.01$ & $<0.01$ & $<0.01$ & $<0.01$ & $<0.01$ & $<0.01$ \\
\hline
\end{tabular}

tissues compared to the CIN1 and normal cervix (Figure 3B, $p<0.01)$.

In this work, the characterization of $\beta$-catenin and coactivator CBP expression implied that the canonical Wnt signaling pathway was activated in high-grade lesions. Consistent with previous studies, CBP-Wnt activity was associated with tumorigenesis and stem cell maintenance because it modulates the levels of intrinsic pluripotency factors, such as Oct4, Nanog, and Sox2 [6]. Here, CK17, a marker of reserve cells and TICs [23, 24], was expressed in the basal compartment of the normal cervix and CIN1 samples, while it displayed diffuse immunoreactivity in the SCC and CIN2/3 samples (Figure 4). On the other hand, the differentiation marker Involucrin was extensively expressed in the upper two-thirds of the squamous epithelium in the normal cervix and CIN1 samples, while minimal immunoreactivity was observed in the CIN2/3 or SCC samples (Figure 4).

\section{Correlation Between the Expression of Various Biomarkers With the Cervical Lesion Grade}

The heatmap, which was made with the total IHC scores of various biomarkers and the cervical lesion grade, apparently displayed the association between them (Figure 5A). Spearman test further verified that the expression of Wnt3a, $\beta$-catenin, CBP, P16, Ki67, and CK17 were positively correlated with the cervical lesion grade $(r=0.679,0.577,0.688,0.898,0.892$, 0.867 , respectively, all $p$ value $<0.01$ ), while the expression of Involucrin was negatively correlated with it $(r=-0.557, p<0.01)$
(Table 2). Taken together, these data suggest that Wnt/ $\beta$-catenin/ CBP is activated in CIN2/3 lesions and SCC, where it maintains TIC pluripotency and promotes cell proliferation, thereby contributing to neoplastic development.

\section{DISCUSSION}

In this report, we described the identification of canonical Wnt signaling activation in precancerous lesions and cervical cancer. Wnt proteins comprise a large family of secreted growth factors that play essential roles in regulating cell proliferation, survival, differentiation, tissue architecture, and organogenesis during embryonic development [6-9]. Accumulating evidence suggests that the Wnt/ $\beta$-catenin signaling pathway serves critical functions in the sequential development of the neoplastic process from initiation, proliferation and transformation in a broad range of cancer types, such as colorectal, breast, and cervical cancer [7, 11, 18, 24-27]. The activation of the canonical Wnt pathway is characterized by the accumulation of $\beta$-catenin in the cytoplasm and nucleus; nevertheless, $\beta$-catenin is mainly located at cellular junctions in the absence of Wnt ligands [28-30].

In this study, the gene expression profile data showed that within the top 250 differentially expressed genes, the canonical Wnt signaling genes Wnt $3 a, \beta$-catenin, and $C B P$ were significantly upregulated in the CIN lesion and cervical cancer samples compared with those in the normal cervix sample. These bioinformatics data were further verified by 
immunohistochemical staining. In particular, a gradual increase in the $\beta$-catenin immunoreactivity score and its positive translocation from the membrane to the cytoplasm and finally to the nucleus were observed in CIN2/3 and SCC, which indicates the activation of Wnt signaling $[6,8,12]$. These excess $\beta$-catenin translocated to the nucleus and activated the downstream genes expression, including c-Myc, Sox2, Nanog, Oct4, Survivin and Cyclin D1, thus contributing to oncogenesis and cancer development $[6,27,31]$.

The physical association between $\beta$-catenin and $\mathrm{CBP} / \mathrm{p} 300$, which are cotranscriptional factors, influences $\mathrm{Wnt} / \beta$-catenin signaling $[6,10,32]$. CBP-mediated Wnt activity is essential for cell proliferation and pluripotency and has a central role in advanced stages of neoplastic development [6, 33], whereas p300/ $\beta$-catenin-mediated transcription is the critical step in initiating differentiation and decreasing cellular potency [10, $32,34]$. The activation of $\mathrm{CBP} / \beta$-catenin is deemed the default active pathway in stem cells, which maintains an undifferentiated proliferative state $[35,36]$. For regular development to proceed, cells must exit the cell cycle and initiate the process of differentiation [37-39]. The array data analysis performed with GEO2R showed that the expression of $C B P / \beta$-catenin and its target genes of KLF4, MYC, and SOX2 were upregulated, generally synchronicity with the progression of cervical lesion grade. Consistent with the activation of CBP/ $\beta$-catenin, markers of TICs CK17, CK14, TP63 and P16 were upregulated, while markers of squamous cell differentiation LORICRIN, INVOLUCRIN and CK4 were downregulated significantly in the CIN and SCC samples compared with normal cervix samples. However, the expression of KLF4 and CK14 were slightly decreased in CIN as compared with normal cervix. We speculated that the lesion grade (CIN1 or CIN2/3) and the status of high-risk HPV infection in CIN samples, to some extent, may interfere the results. The immunoreactivity scores analyzed with Spearman test showed that the expression of Ki67, P16, CK17 were positively correlated with the cervical lesion degree while Involucrin was just the opposite. Thus, both array and IHC data further verified that canonical Wnt signaling activation promotes cervical lesion progression, which indicates the potential role of Wnt3a in maintaining stemness of TICs reprogrammed by Piwil2 in our previous report and sequentially triggering the progression of $\mathrm{CIN} 2 / 3$ and SCC [4]. Furthermore, inhibiting the $\mathrm{CBP} / \beta$-catenin interaction with specific antagonists (i.e., ICG-001 or PRI724) led to symmetric CSC divisions at the expense of asymmetric divisions; thus, CSCs are stochastically cleared from their niche via symmetric differentiative division [40-42]. Therefore, selectively antagonizing the CBP/ $\beta$-catenin interaction may be a potential target for $\mathrm{CIN} 2 / 3$ and SCC therapy.

\section{REFERENCES}

1. Chibwesha CJ, Stringer JSA. Cervical cancer as a global concern. JAMA (2019) 322(16):1558-60. doi:10.1001/jama.2019.16176
In conclusion, our observations demonstrate that the canonical Wnt signaling pathway was activated during the oncogenesis of cervical lesions. The $\mathrm{CBP} / \beta$-catenin interaction played an essential role in the progression of CIN and SCC by maintaining the stemness of TICs (Figure 5B). Therefore, targeting the $\mathrm{CBP} / \beta$-catenin interaction may be a promising therapeutic strategy for preventing CIN progression.

\section{DATA AVAILABILITY STATEMENT}

The datasets presented in this study can be found in online repositories. The names of the repository/repositories and accession number(s) can be found in the article/ Supplementary Material.

\section{ETHICS STATEMENT}

The studies involving human participants were reviewed and approved by the Ethics Committee of China-Japan Friendship Hospital (approval number: 2020-28-K20). Written informed consent for participation was not required for this study in accordance with the national legislation and the institutional requirements.

\section{AUTHOR CONTRIBUTIONS}

DQF performed bioinformatics analysis. JL, WHW, HYL, JL, and $\mathrm{HY}$ carried out immunohistochemistry staining. KQY and BL analyzed the experimental results. All authors wrote and revised the manusript.

\section{FUNDING}

This work was supported by the National Key R\&D Program of China (2018YFC1003900), the National Natural Science Foundation of China (81372777, 81372779), and the Fundamental Research Funds for the Central Universities and Research projects on biomedical transformation of China-Japan Friendship Hospital (No. PYBZ1827).

\section{CONFLICT OF INTEREST}

The authors declare that the research was conducted in the absence of any commercial or financial relationships that could be construed as a potential conflict of interest.

2. Chen W, Zheng R, Baade PD, Zhang S, Zeng H, Bray F, et al. Cancer statistics in China, 2015. CA: A Cancer J Clinicians (2016) 66(2):115-32. doi:10.3322/caac. 21338

3. Ferenczy A, Franco E. Persistent human papillomavirus infection and cervical neoplasia. Lancet Oncol (2002) 3(1):11-6. doi:10.1016/s1470-2045(01)00617-9 
4. Feng D, Yan K, Zhou Y, Liang H, Liang J, Zhao W, et al. Piwil2 is reactivated by HPV oncoproteins and initiates cell reprogramming via epigenetic regulation during cervical cancer tumorigenesis. Oncotarget (2016) 7(40):64575-88. doi:10.18632/oncotarget.11810

5. Pakula H, Xiang D, Li Z. A tale of two signals: AR and WNT in development and tumorigenesis of prostate and mammary gland. Cancers (2017) 9(2):14. doi:10. 3390/cancers 9020014

6. Kahn M. Wnt signaling in stem cells and cancer stem cells: a tale of two coactivators. Prog Mol Biol Transl Sci (2018) 153:209-44. doi:10.1016/bs.pmbts.2017.11.007

7. Yan KS, Janda CY, Chang J, Zheng GXY, Larkin KA, Luca VC, et al. Nonequivalence of Wnt and R-spondin ligands during Lgr5+ intestinal stem-cell self-renewal. Nature (2017) 545(7653):238-42. doi:10.1038/nature22313

8. Doumpas N, Lampart F, Robinson MD, Lentini A, Nestor CE, Cantù C, et al. TCF/LEF dependent and independent transcriptional regulation of Wnt/ $\beta$-catenin target genes. EMBO J (2019) 38(2). doi:10.15252/embj.201798873

9. Lukaszewicz AI, Nguyen C, Melendez E, Lin DP, Teo J-L, Lai KKY, et al. The mode of stem cell division is dependent on the differential interaction of $\beta$-catenin with the Kat3 coactivators CBP or p300. Cancers (2019) 11(7):962. doi:10.3390/cancers 11070962

10. Rieger ME, Zhou B, Solomon N, Sunohara M, Li C, Nguyen C, et al. p300/ $\beta$-Catenin interactions regulate adult progenitor cell differentiation downstream of WNT5a/protein kinase C (PKC). J Biol Chem (2016) 291(12):6569-82. doi:10.1074/jbc.M115.706416

11. Hoffmeyer K, Raggioli A, Rudloff S, Anton R, Hierholzer A, Del Valle I, et al. Wnt/-catenin signaling regulates telomerase in stem cells and cancer cells. Science (2012) 336(6088):1549-54. doi:10.1126/science.1218370

12. Schaefer KN, Peifer M. Wnt/Beta-catenin signaling regulation and a role for biomolecular condensates. Develop Cel (2019) 48(4):429-44. doi:10.1016/j. devcel.2019.01.025

13. Martins-Neves SR, Corver WE, Paiva-Oliveira DI, van den Akker BEWM, Briaire-de-Bruijn IH, Bovée JVMG, et al. Osteosarcoma stem cells have active wnt/ $\beta$-catenin and overexpress SOX2 and KLF4. J Cel Physiol (2016) 231(4): 876-86. doi:10.1002/jcp.25179

14. Trisno SL, Philo KED, McCracken KW, Catá EM, Ruiz-Torres S, Rankin SA, et al. Esophageal organoids from human pluripotent stem cells delineate Sox2 functions during esophageal specification. Cell Stem Cell (2018) 23(4):501-15. doi:10.1016/j.stem.2018.08.008

15. Yong X, Tang B, Xiao Y-F, Xie R, Qin Y, Luo G, et al. Helicobacter pylori upregulates Nanog and Oct4 via Wnt/ $\beta$-catenin signaling pathway to promote cancer stem cell-like properties in human gastric cancer. Cancer Lett (2016) 374(2):292-303. doi:10.1016/j.canlet.2016.02.032

16. Ogawa K, Nishinakamura R, Iwamatsu Y, Shimosato D, Niwa H. Synergistic action of Wnt and LIF in maintaining pluripotency of mouse ES cells. Biochem Biophysical Res Commun (2006) 343(1):159-66. doi:10.1016/j.bbrc.2006.02.127

17. Zandi M, Muzaffar M, Shah SM, Kaushik R, Singh MK, Palta P, et al. WNT3A signalling pathway in buffalo (Bubalus bubalis) embryonic stem cells. Reprod Fertil Dev (2014) 26(4):551-61. doi:10.1071/RD13084

18. Nourbakhsh M, Mansoor A, Koro K, Zhang Q, Minoo P. Expression profiling reveals involvement of WNT pathway in the malignant progression of sessile serrated adenomas. Am J Pathol (2019) 189(9):1732-43. doi:10.1016/j.ajpath.2019.05.009

19. Lane TF, Leder P. Wnt-10b directs hypermorphic development and transformation in mammary glands of male and female mice. Oncogene (1997) 15(18):2133-44. doi:10.1038/sj.onc.1201593

20. Wong GT, Gavin BJ, McMahon AP. Differential transformation of mammary epithelial cells by Wnt genes. Mol Cel Biol. (1994) 14(9):6278-86. doi:10.1128/ mcb.14.9.6278

21. Zhuang S-M, Wiseman RW, Söderkvist P. Frequent mutations of the Trp53, Hras1 and $\beta$-catenin (Catnb) genes in 1,3-butadiene-induced mammary adenocarcinomas in B6C3F1 mice. Oncogene (2002) 21(36):5643-8. doi:10.1038/sj.onc.1205649

22. Moser AR, Mattes EM, Dove WF, Lindstrom MJ, Haag JD, Gould MN. ApcMin, a mutation in the murine Apc gene, predisposes to mammary carcinomas and focal alveolar hyperplasias. Proc Natl Acad Sci (1993) 90(19):8977-81. doi:10.1073/pnas.90.19.8977

23. Martens JE, Arends J, Van der Linden PJ, De Boer BA, Helmerhorst TJ. Cytokeratin 17 and p63 are markers of the HPV target cell, the cervical stem cell. Anticancer Res (2004) 24(2B):771-5.

24. Ortiz-Sánchez E, Santiago-López L, Cruz-Domínguez VB, Toledo-Guzmán ME, Hernández-Cueto D, Muñiz-Hernández S, et al. Characterization of cervical cancer stem cell-like cells: phenotyping, stemness, and human papilloma virus co-receptor expression. Oncotarget (2016) 7(22):31943-54. doi:10.18632/oncotarget.8218

25. Zhan T, Ambrosi G, Wandmacher AM, Rauscher B, Betge J, Rindtorff N, et al. MEK inhibitors activate Wnt signalling and induce stem cell plasticity in colorectal cancer. Nat Commun (2019) 10(1):2197. doi:10.1038/s41467-019-09898-0

26. Liu X-F, Li X-Y, Zheng P-S, Yang W-T. DAX1 promotes cervical cancer cell growth and tumorigenicity through activation of $\mathrm{Wnt} / \beta$-catenin pathway via GSK3 $\beta$. Cell Death Dis (2018) 9(3):339. doi:10.1038/s41419-018-0359-6

27. Dai Y, Liu L, Zeng T, Liang J-Z, Song Y, Chen K, et al. Overexpression of MUC13, a poor prognostic predictor, promotes cell growth by activating Wnt signaling in hepatocellular carcinoma. Am J Pathol (2018) 188(2):378-91. doi:10.1016/j.ajpath.2017.10.016

28. Ayala-Calvillo E, Mojica-Vázquez LH, García-Carrancá A, González-Maya L. Wnt/ $\beta$ catenin pathway activation and silencing of the APC gene in HPVpositive human cervical cancerderived cells. Mol Med Rep (2018) 17(1):200-8. doi:10.3892/mmr.2017.7853

29. Kim B, Byun S-J, Kim YA, Kim JE, Lee BL, Kim WH, et al. Cell cycle regulators, $\mathrm{APC} / \beta$-catenin, NF- $\mathrm{KB}$ and Epstein-Barr virus in gastric carcinomas. Pathology (2010) 42(1):58-65. doi:10.3109/00313020903356392

30. Üren A, Fallen S, Yuan H, Usubütün A, Küçükali T, Schlegel R, et al. Activation of the canonical Wnt pathway during genital keratinocyte transformation: a model for cervical cancer progression. Cancer Res (2005) 65(14):6199-206. doi:10.1158/0008-5472.CAN-05-0455

31. Gang EJ, Hsieh Y-T, Pham J, Zhao Y, Nguyen C, Huantes S, et al. Smallmolecule inhibition of $\mathrm{CBP} /$ catenin interactions eliminates drug-resistant clones in acute lymphoblastic leukemia. Oncogene (2014) 33(17):2169-78. doi:10.1038/onc.2013.169

32. Li J, Sutter C, Parker DS, Blauwkamp T, Fang M, Cadigan KM. CBP/p300 are bimodal regulators of Wnt signaling. EMBO J (2007) 26(9):2284-94. doi:10. 1038/sj.emboj.7601667

33. Bordonaro M, Lazarova DL. CREB-binding protein, p300, butyrate, and Wnt signaling in colorectal cancer. $W j g$ (2015) 21(27):8238-48. doi:10.3748/wjg. v21.i27.8238

34. Bordonaro M, Lazarova DL. Determination of the role of CBP- and p300mediated Wnt signaling on colonic cells. JMIR Res Protoc (2016) 5(2):e66. doi:10.2196/resprot.5495

35. Ring A, Kim Y-M, Kahn M. Wnt/catenin signaling in adult stem cell physiology and disease. Stem Cel Rev Rep (2014) 10(4):512-25. doi:10.1007/s12015-014-9515-2

36. Malleske DT, Hayes D, Jr, Lallier SW, Hill CL, Reynolds SD. Regulation of human airway epithelial tissue stem cell differentiation by $\beta$-catenin, P300, and CBP. Stem Cells (2018) 36(12):1905-16. doi:10.1002/stem.2906

37. Jakoby M, Schnittger A. Cell cycle and differentiation. Curr Opin Plant Biol (2004) 7(6):661-9. doi:10.1016/j.pbi.2004.09.015

38. Otto T, Candido SV, Pilarz MS, Sicinska E, Bronson RT, Bowden M, et al. Cell cycle-targeting microRNAs promote differentiation by enforcing cell-cycle exit. Proc Natl Acad Sci USA (2017) 114(40):10660-5. doi:10.1073/pnas.1702914114

39. Wang Y, Zhao J, Cao C, Yan Y, Chen J, Feng F, et al. The role of E2F1-topoII $\beta$ signaling in regulation of cell cycle exit and neuronal differentiation of human SH-SY5Y cells. Differentiation (2018) 104:1-12. doi:10.1016/j.diff.2018.07.002

40. Kahn M. Symmetric division versus asymmetric division: a tale of two coactivators. Future Med Chem (2011) 3(14):1745-63. doi:10.4155/fmc.11.126

41. Tang Y, Berlind J, Mavila N. Inhibition of CREB binding protein-beta-catenin signaling down regulates CD133 expression and activates PP2A-PTEN signaling in tumor initiating liver cancer cells. Cell Commun Signal (2018) 16(1):9. doi:10.1186/s12964-018-0222-5

42. Chan KC, Chan LS, Ip JCY, Lo C, Yip TTC, Ngan RKC, et al. Therapeutic targeting of $\mathrm{CBP} / \beta$-catenin signaling reduces cancer stem-like population and synergistically suppresses growth of EBV-positive nasopharyngeal carcinoma cells with cisplatin. Sci Rep (2015) 5:9979. doi:10.1038/srep09979

Copyright $\odot 2021$ Feng, Lin, Wang, Yan, Liang, Liang, Yu and Ling. This is an openaccess article distributed under the terms of the Creative Commons Attribution License (CC BY). The use, distribution or reproduction in other forums is permitted, provided the original author(s) and the copyright owner(s) are credited and that the original publication in this journal is cited, in accordance with accepted academic practice. No use, distribution or reproduction is permitted which does not comply with these terms. 